\title{
Dosificación de digoxina: lo habitual y lo óptimo
}

Conthe P, Montoto C. Dosificación de digoxina: lo habitual y lo óptimo. An Med Interna (Madrid) 2002; 19: 329-330.

La digoxina constituye uno de los fármacos clásicos en el tratamiento de la insuficiencia cardiaca. Ya en 1785 Whithering escribió sobre el uso de la digoxina en un clásico tratado (1) que contenía la primera descripción de los glicósidos digitalicos en el tratamiento de la insuficiencia cardiaca congestiva. Este tratado describía la eficacia y toxicidad de las hojas de una planta común, la Digitalis purpúrea para mejorar los síntomas de esta patología. Su uso ha fluctuado a lo largo de la historia debido a los controvertidos datos sobre su utilidad y ha llegado a estar en entre dicho en ciertos momentos ante los controvertidos resultados obtenidos con algunos otros inotrópicos (2).

Aunque se utiliza este fármaco desde hace más de dos siglos, aún se discute el mecanismo de acción en la insuficiencia cardiaca. Los glucósidos cardiotónicos actúan de manera directa sobre el miocardio, donde incrementan la contractilidad intrínseca de la fibra muscular (efecto inotrópico positivo) mediante la saturación reversible y de alta afinidad a sitios específicos que forman parte de la enzima ATPasa- $\mathrm{N}+/ \mathrm{K}+$ dependiente. Este efecto determina un incremento en la velocidad y amplitud del acortamiento del músculo cardiaco, tanto en el miocardio normal como en el alterado y tanto en el músculo ventricular como en el auricular. Este efecto se mantiene durante meses sin evidencias de desensibilización o tolerancia (3). Sin embargo algunas observaciones hacen creer hoy en día que el efecto en la insuficiencia cardiaca procede más de efectos indirectos que modifican los mecanismos compensadores neuroendocrinos así como el tono simpático. Se sabe que la insufiencia cardiaca se acompaña a menudo de un incremento de la actividad del sistema nervioso simpático debido en parte a la reducción en la sensibilidad de la respuesta arterial a la tensión arterial. El tratamiento con digoxina parece modificar esta situación como demostró un estudio prospectivo en 26 pacientes ambulatorios con grados variables de insuficiencia cardiaca (clase funcional NYHA de I a III), en los que los niveles de norepinefrina plasmática descendieron significativamente. El análisis de la variabilidad de la frecuencia cardiaca antes y después de tratamiento crónico con digoxina reveló un aumento en la actividad del parasimpático, con una considerable reducción de la actividad del sistema nervioso simpático (4).

Muchas han sido las modificaciones que ha sufrido el tratamiento de la insuficiencia cardiaca por disfunción sistólica en los últimos años en un intento de mejorar el pésimo pronóstico de estos pacientes (5). Del simple esquema de la restricción de ejercicio, la digoxina y los diuréticos se ha pasado a un auténtico arsenal terapéutico, que aun así no ha mejorado notablemente el pronóstico. El éxito conseguido en el pronóstico de pacientes con disfunción sistólica con los inhibidores del enzima convertidor de angiotensina (IECAs), ha puesto en evidencia la importancia del bloqueo neurohumoral y de cómo bloqueando otros sistemas hormonales se puede obtener un efecto aditivo, aspecto en el que se centra actualmente la terapia de la IC. En esta línea se ha desarrollado la investigación en este campo con la inclusión de otros fármacos como la espironolactona o los beta-bloqueantes en el tratamiento de esta prevalente patología. Estas novedades en el esquema terapéutico y los decepcionantes resultados obtenidos con algunos fármacos inotrópicos positivos, dejó de alguna manera, relegada a un segundo plano a la digoxina. Ya en el estudio RADIANCE se había encontrado como la digoxina mejoraba la capacidad funcional y se reducían los síntomas en la mayoría de los pacientes. Además en aquellos pacientes que estaban siendo tratados con digoxina, diuréticos y un IECA, y la digoxina era suspendida, la probabilidad de presentar un deterioro clínico era de seis veces mayor respecto a los que no la dejaban de tomar (6). Pero hasta ese momento no existía ningún ensayo con población suficiente para determinar si la digoxina afectaba a la mortalidad de los pacientes con insuficiencia cardíaca. Desde la publicación de los resultados del estudio DIG (7) este fármaco ha recuperado un papel claro en ciertos aspectos del tratamiento de la IC. En este estudio se demostró sobre un amplio número de pacientes con IC en ritmo sinusal, el efecto neutro sobre la mortalidad del tratamiento con digoxina (con este fármaco se produce una disminución del riesgo de muerte en insuficiencia cardiaca pero con un pequeño aumento del número de infartos de miocardio y muertes súbitas). Se consiguió sin embargo una disminución significativa de los ingresos hospitalarios en el grupo con digoxina $(\mathrm{p}<0,001)$. Se podía concluir que la digoxina supone hoy un fármaco con un leve efecto de mejoría del estado sintomático, disminuyendo el riesgo de hospitalización sin afectar a la supervivencia de los pacientes. Por ello la digoxina aparece hoy en día con unas indicaciones claras en las guías de actuación en Insuficiencia Cardiaca de las más prestigiosas sociedades científicas. En ellas se recoge su indicación como fármaco para control de la fibrilación auricular en cualquier grado de insuficiencia cardiaca sintomática, y en los pacientes en ritmo sinusal, para mejorar el 
estado clínico en caso de síntomas debidos a disfunción sistólica ventricular izquierda, cuando estos persisten a pesar del tratamiento con IECAS y diuréticos a dosis adecuadas (8).

La familiaridad del clínico con este fármaco sin embargo es alta, a juzgar por los datos de su uso en grandes registros de pacientes con IC. Tanto en el publicado por Cohen-Sola en Francia (9) como en el del Grupo de Trabajo de la Sociedad Española de Medicina Interna (10), la digoxina es el tercer fármaco más utilizado en el tratamiento de estos pacientes por detrás de los diuréticos y los IECAS y muy por delante de los betabloqueantes. A pesar de este uso habitual de la digoxina, las intoxicaciones siguen siendo frecuentes en la práctica clínica, si bien su incidencia y severidad han disminuido (11). Hoy en día los esquemas de dosificación, si bien no son rígidos permiten hablar de pautas generales que se alejan de los "descansos" de fin de semana tradicionales en la administración de los preparados antiguos, recordando que en el control de la fibrilación auricular se precisan dosis más altas. Sin embargo la vigilancia de la intoxicación digitálica, importante complicación de la terapia, es esencial. Aunque existen normogramas disponibles para la estimación de las dosis de carga y mantenimiento de digoxina su uso no está muy extendido por la variabilidad en la respuesta individual a los glicósidos cardiacos. Exinten varios factores que influyen en la sensibilidad de los pacientes a la digoxina. Además de la edad y la función renal, otros determinan una modificación en la sensibilidad al fármaco (alteraciones hidroelectrolíticas, alteraciones en función tiroidea, interacciones con ciertos fármacos...), pero cómo inciden estos en un paciente concreto es a veces difícil de determinar a priori. Esto complica los ajustes de dosis en los pacientes en los que se utiliza este fármaco, que son obviamente pacientes con insuficiencia cardiaca en los que concurren con frecuencia muchos de estos factores dada su alta comorbilidad y el gran número de fármacos que tienen en su esquema terapeutico. De ahí la clásica búsqueda de modelos matemáticos que permitan un adecuado ajuste de dosis teniendo en cuenta al menos algunos de los principales parámetros del paciente, minimizando así el riesgo de toxicidad.

Para conseguir rangos adecuados que permitan en un pacien- te un efecto terapéutico y dificulten la presencia de toxicidad grave, Cuena Boy y colaboradores (12), intentan establecer el mejor método de ajuste de dosis, comparando los más contrastados. En 87 pacientes se evaluó el rendimiento del método Lin comparado con el Jellife y una variante de este último que tiene en cuenta que el volumen de distribución del fármaco depende del aclaramiento de creatinina . Estos métodos (poco conocidos en la práctica clínica diaria y más familiares para los farmacólogos) permiten el ajuste de dosis en la mayoría de los pacientes en rangos entre 0,8 y 2,0 ng/ml, aunque el método Lin parece permitir mejores resultados y una mayor precisión. Establecer la utilidad real de este método en el ajuste de digoxina en pacientes ancianos (cuando no se pretende reducir urgentemente la frecuencia cardiaca y siempre que no concurran circunstancias como las que se citaron) no es sin embargo tarea fácil. Estos métodos no evitan los controles analíticos iniciales ni la posibilidad de la intoxicación en ciertas circunstancias, pero sí permiten un mejor ajuste determinando el momento aproximado en el que se alcanza el efecto definitivo y por tanto como programar mejor los controles. Por otro lado permite evitar las dosis infraterapeuticas (tan frecuentes en la práctica clínica) aparecidas al no utilizar métodos de ajuste de dosis. Asimismo evita comenzar siempre por dosis bajas que retrasarán el control sintomático del paciente.

Aunque, de potencial utilidad, la escasa familiaridad del clínico con las fórmulas propuestas en la práctica diaria hace que este encuentre difícil la aplicación del método en la clínica. Sin embargo se debería reflexionar sobre la posible utilidad de dedicar unos minutos a este método que se ofrece como herramienta útil en el ajuste de un fármaco de eficacia probada para evitar la aparición de situaciones clínicas graves y optimizar su rendimiento terapeutico.

\section{P. CONTHE, C. MONTOTO}

Servicio de Medicina Interna I. Hospital General Universitario Gregorio Marañón. Madrid

\section{Bibliografía}

1. Withering W. An account of the fox glove and some of its medical uses: With practical remarks on dropsy and other diseases. Med Classics 1937; 2: 295-443

2. Ewy GA. Inotropic infusions for chronic congestive heart failure. Medical miracles or misguided medicinals? J Am Coll Cardiol 1999; 33: 572-575.

3. Schmidt TA, Allen PD, Colucci WS. No adaptation to digitalization as evaluated by digitalis receptor ( $\mathrm{Na}, \mathrm{K}$ - ATPase) quantification in explanted hearts from donors without heart disease and from digitalized recipients with end-stage heart failure. Am J Cardiol 1992; 70: 110114.

4. Krum H, Bigger JT, Goldsmith RL. Effect of long-term digoxin therapy on autonomic function in patients with chronic heart failure. J Am Coll Cardiol 1995; 25: 289-292.

5. Cowie MR, Mosterd A, Wood DA, Deckers JW, Poole-Wilson PA, Sutton GC et al. The epidemiology of heart failure. Eur Heart J 1997; 18 : 208- 225 .

6. Packer M, Gheorghiade M, Young JB. Withdrawal of digoxin from patients with chronic heart failure treated with angiotensing converting enzyme inhibitors. RADIANCE study. N Engl J Med 1993; 329: 1-7.

7. The Digitalis Investigation Group. The effect of digoxin on mortality and morbidity in patients with Heart Failure. N Engl J Med 1997; 336: 525-533.

8. Task Force for the Diagnosis and Treatment of Chronic Heart Failure, European Society of Cardiology. Guidelines for the diagnosis and treatment of chronic heart failure. Eur Heart J 2001; 22: 1527-1560.

9. Cohen-Sola A, Desnos M, Delahaye F, Emeriau JP, Hanania G. A national survey of heart failure in French hospitals. Eur Heart J 2000; 21: 763-769.

10. Grupo de Trabajo de IC de la Sociedad Española de Medicina Interna. La insuficiencia cardiaca en los servicios de Medicina Interna. Med Clin (Barcelona) 2002; 118 (16): 605-10.

11. Mahdyoon H, Battilana G, Rosman H. The evolving pattern of digoxin intoxication: Observations at a large urban hospital from 1980 to 1988. Am Heart J 1990;120: 1189-93.

12. R Cuena Boy, A Ortiz de Apodaca, MA Maciá Martinez. Mejor resultado del ajuste de la dosis de digoxina en ancianos si se tiene en cuenta que tanto la eliminación como el volumen de distribución del fármaco disminuyen cuando se reduce la función renal. An Med Interna (Madrid). 2002; 19: 331-335 